\title{
Theory of Evaporative Cooling with Energy-Dependent Elastic Scattering Cross Section and Application to Metastable Helium
}

\author{
Paul J. J. Tol, Wim Hogervorst and Wim Vassen \\ Laser Centre Vrije Universiteit, De Boelelaan 1081, 1081 HV Amsterdam, The Netherlands
}

(Dated: June 14, 2018)

\begin{abstract}
The kinetic theory of evaporative cooling developed by Luiten et al. [Phys. Rev. A 53, 381 (1996)] is extended to include the dependence of the elastic scattering cross section on collision energy. We introduce a simple approximation by which the transition range between the low-temperature limit and the unitarity limit is described as well. Applying the modified theory to our measurements on evaporative cooling of metastable helium we find a scattering length $|a|=10(5) \mathrm{nm}$.
\end{abstract}

PACS numbers: 51.10.+y, 05.30.Jp, 32.80.Pj

\section{INTRODUCTION}

Evaporative cooling is at present the most powerful method to increase the phase-space density of a dilute trapped gas in order to reach Bose-Einstein condensation (BEC) or Fermi degeneracy [1]. The atoms are first laser-cooled to temperatures of $1 \mathrm{mK}$ or lower and trapped either magnetically in a Ioffe quadrupole (IQ) trap, or optically in a dipole trap. Here the energy of the atoms is redistributed by elastic collisions. A few atoms acquiring energies larger than the trap depth $\varepsilon_{\mathrm{t}}$ (with a maximum energy of $2 \varepsilon_{\mathrm{t}}$ ) are expelled from the trap. This reduces significantly the mean energy of the trapped ensemble while only a relatively small number of atoms is lost. In forced evaporative cooling the trap depth is gradually lowered, during which cooling increases the density and the elastic collision rate, accelerating evaporation.

In the Walraven group kinetic theory has been developed to describe this evaporative cooling process for cold atomic gases [2, 3]. The theory assumes collisions occurring in the s-wave limit, an energy-independent elastic scattering cross section $\sigma$ and sufficient ergodicity in the trap. A truncated Boltzmann distribution is introduced and shown to be consistent with the classical Boltzmann equation applied to a trap of finite depth. Then equations of statistical mechanics are obtained describing the kinetics, particle loss and energy loss. Closed expressions are derived that are directly applicable to an IQ trap. However, the assumption of an energy-independent cross section $\sigma$ is not justified when evaporation starts in the transition range between the low-temperature limit and the unitarity limit. This is the case, for instance, in metastable helium, where evaporative cooling typically starts at $\sim 1 \mathrm{mK}[4$, , 5 , 6$]$. In order to understand evaporative cooling under these conditions as well, this paper extends the theory to incorporate an energy-dependent $\sigma$. The theory is subsequently applied to interpret our evaporative cooling experiments with metastable helium, allowing the s-wave scattering length $a$ to be extracted. As BEC has been realized by two groups in France [4, 5], $a$ is known to be large and positive. Published experimental values are $a=+20(10) \mathrm{nm}[4]$ and $a=+16(8) \mathrm{nm}$ [5], both obtained from Bose-condensed clouds. Recent experiments in Paris 7] with clouds just above the BEC transition suggest that $a$ is smaller than previously published values. Theoretical values determined from precisely calculated molecular potentials are $+8.3 \mathrm{~nm}[8]$ and $+12.2 \mathrm{~nm}[9,10]$.

This paper is organized as follows. In Section $\Pi$ the theory of evaporative cooling based on the papers of Luiten et al. 2] and Pinkse et al. [3] is summarized and extended. First, Section IA gives the density and energy of an atomic cloud in a magnetic trap of finite depth and Section [B provides the equations necessary to calculate all relevant loss rates during the cooling process, assuming $\sigma$ is energy-independent. Then, in Section IIC the atom and energy loss rates due to evaporation are recalculated, now with an energy-dependent cross section $\sigma$. Additionally, a simple approximate expression is presented for an effective cross section that can be implemented directly in the equations for the evaporation loss rates given before in Section [B] The theory is used in Section III to interpret evaporative cooling experiments in metastable helium performed in Amsterdam and a value for the scattering length is deduced. Section [V] summarizes and compares the extracted scattering length with results obtained in the other experiments and with theoretical values from molecular potential calculations.

\section{THEORY}

\section{A. Density in magnetic traps with finite depth}

The trapping potential due to a magnetic field $B(\boldsymbol{r})$ with (local) minimum $B_{0}$ is given by $U(\boldsymbol{r})=m_{J} g_{J} \mu_{\mathrm{B}}\left[B(\boldsymbol{r})-B_{0}\right]$, where $g_{J}$ is the Landé $g$-factor and $m_{J}$ the magnetic quantum number of the state of the trapped atoms. We use 
metastable helium in the $2{ }^{3} \mathrm{~S}_{1}, m_{J}=1$ state with $g_{J} \approx 2$. The trapping potential of an IQ trap can be approximated by

$$
U(x, y, z)=\sqrt{\alpha^{2}\left(x^{2}+y^{2}\right)+\left(U_{0}+\beta z^{2}\right)^{2}}-U_{0},
$$

with minimum potential energy

$$
U_{0}=m_{J} g_{J} \mu_{\mathrm{B}} B_{0}
$$

where the effect of gravity is neglected.

According to Luiten et al. 2] the atoms in a magnetic trap with a finite depth are described well by a Boltzmann energy distribution that is truncated at an energy equal to the trap depth. The phase-space distribution is assumed to be only a function of the energy of the atoms. At low temperatures this is the case if there are many elastic collisions between the atoms. At higher temperatures in the IQ trap, the atoms occupy a part of space large enough that higher-order terms of the potential break the axial symmetry of Eq. (11) and the motion of the atoms becomes ergodic even without collisions. Below a summary is given of the equations needed to calculate the density distribution in a magnetic trap [2].

The (thermal) density distribution in an infinitely deep trap is

$$
n_{\infty}(\boldsymbol{r})=n_{0} \exp \left[-\frac{U(\boldsymbol{r})}{k_{\mathrm{B}} T}\right] .
$$

When only atoms with an energy smaller than $\varepsilon_{\mathrm{t}}$ are trapped, the density distribution becomes

$$
n(\boldsymbol{r})=\mathrm{P}\left[\frac{3}{2}, \kappa(\boldsymbol{r})\right] n_{\infty}(\boldsymbol{r}),
$$

with incomplete gamma function $\mathrm{P}[11]$ and

$$
\kappa(\boldsymbol{r})= \begin{cases}\frac{\varepsilon_{\mathrm{t}}-U(\boldsymbol{r})}{k_{\mathrm{B}} T}, & U(\boldsymbol{r}) \leq \varepsilon_{\mathrm{t}}, \\ 0, & U(\boldsymbol{r})>\varepsilon_{\mathrm{t}} .\end{cases}
$$

In this truncated Boltzmann distribution $T$ is still called the temperature, although strictly speaking a thermodynamic temperature is not defined for a nonequilibrium distribution. The central density is given by $n(0)=\mathrm{P}\left[\frac{3}{2}, \kappa(\boldsymbol{r})\right] n_{0}$; parameter $n_{0}$ is equal to the central density only in the limit of infinite trap depth. It is defined by

$$
n_{0}=N / V_{\mathrm{e}}
$$

with number of trapped atoms $N$ and reference volume $V_{\mathrm{e}}$, which is equal to the effective volume $N / n(0)$ in the limit of a deep trap. In general the reference volume is given by

$$
V_{\mathrm{e}}=\Lambda^{3} \zeta
$$

with thermal de Broglie wavelength

$$
\Lambda=\sqrt{\frac{2 \pi \hbar^{2}}{m k_{\mathrm{B}} T}}
$$

and trap-dependent single-atom partition function $\zeta$. For an IQ trap

$$
\begin{aligned}
& \zeta=\zeta_{\infty}^{0}\left[\mathrm{P}(4, \eta)+\frac{2}{3} \frac{U_{0}}{k_{\mathrm{B}} T} \mathrm{P}(3, \eta)\right] \\
& \zeta_{\infty}^{0}=6 A_{\mathrm{IQ}}\left(k_{\mathrm{B}} T\right)^{4} \\
& A_{\mathrm{IQ}}=m^{3 / 2} /\left(4 \sqrt{2 \beta} \alpha^{2} \hbar^{3}\right)
\end{aligned}
$$

with truncation parameter

$$
\eta=\frac{\varepsilon_{\mathrm{t}}}{k_{\mathrm{B}} T}
$$

The total internal energy of the trapped atoms is

$$
E=\frac{12 \mathrm{P}(5, \eta)+6 \frac{U_{0}}{k_{\mathrm{B}} T} \mathrm{P}(4, \eta)}{3 \mathrm{P}(4, \eta)+2 \frac{U_{0}}{k_{\mathrm{B}} T} \mathrm{P}(3, \eta)} N k_{\mathrm{B}} T .
$$

Although $n_{0}$ is not the central density and a thermodynamic temperature cannot be given for this nonequilibrium distribution, the (central) phase-space density (or degeneracy parameter) is still $n_{0} \Lambda^{3}$. 


\section{B. Evaporative cooling with constant cross section}

The behavior of a cloud of atoms during an rf sweep is simulated with the model of Luiten et al. [2] and Pinkse et al. 3]. The version of the model described here assumes that every atom with an energy greater than the trap depth $\varepsilon_{\mathrm{t}}$ leaves the trap (three-dimensional evaporation). Their trajectories should bring these atoms sufficiently fast to the exit area of the trap (one of the saddle points or, when an rf field is applied, positions where the rf field is resonant with the magnetic field), so they are removed without first colliding with another atom. Another assumption is that collisions occur in the s-wave regime.

At the start, $N$ atoms at temperature $T$ are contained in a trap with depth $\varepsilon_{\mathrm{t}}$ determined by the magnetic field configuration. After a time step of negligible size the rf power is turned on: the rf frequency $\omega_{\mathrm{rf}}$, the time dependence of which is known beforehand, determines the truncation energy $\varepsilon_{\mathrm{t}}=\hbar \omega_{\mathrm{rf}}-U_{0}$. Then in small steps (for our situation $10 \mathrm{~ms}$ ) the loss of atoms and energy due to inelastic collisions, trap changes (spilling) and evaporation is determined, as discussed below. The temperature is found by solving numerically the equation for the total energy of the trapped atoms, Eq. (11). If experimentally an extra temperature increase is found, for instance due to instability of the power supplies, this can be added separately. The calculation is stopped when either no atoms are left or when $\mathrm{BEC}$ is reached. In the last case the phase-space density is $n_{0} \Lambda^{3} \geq g_{3 / 2}(1) \approx 2.6$, with polylogarithm function $g_{n}(z)=\sum_{k=1}^{\infty} z^{k} / k^{n}$.

References [2, 3] do not give all loss rates in a suitable form, so first some intermediate equations are given and the energy density is derived. Trapped atoms have energy

$$
\varepsilon(\boldsymbol{r}, \boldsymbol{p})=U(\boldsymbol{r})+p^{2} / 2 m
$$

The number of atoms with energy between $\varepsilon$ and $\varepsilon+\mathrm{d} \varepsilon$ is $\rho(\varepsilon) f(\varepsilon) \mathrm{d} \varepsilon$, with phase-space distribution

$$
f(\varepsilon)=n_{0} \Lambda^{3} \exp \left(-\frac{\varepsilon}{k_{\mathrm{B}} T}\right)
$$

and (in an IQ trap) an energy density of states

$$
\rho(\varepsilon)=A_{\mathrm{IQ}}\left(\varepsilon^{3}+2 U_{0} \varepsilon^{2}\right)
$$

The phase-space distribution can be given as a function of $\boldsymbol{r}$ and $\boldsymbol{p}$ via Eq. (12) and is normalized so that the total number of trapped atoms is

$$
N=\frac{1}{(2 \pi \hbar)^{3}} \iint f(\boldsymbol{r}, \boldsymbol{p}) \mathrm{d}^{3} r \mathrm{~d}^{3} p,
$$

where the integration is done over the volume in phase space where $\varepsilon \leq \varepsilon_{\mathrm{t}}$. The density is given by

$$
\begin{aligned}
n(\boldsymbol{r}) & =\frac{1}{(2 \pi \hbar)^{3}} \int f(\boldsymbol{r}, \boldsymbol{p}) \mathrm{d}^{3} p \\
& =\frac{n_{0} \Lambda^{3}}{(2 \pi \hbar)^{3}} \int_{0}^{\sqrt{2 m\left[\varepsilon_{\mathrm{t}}-U(\boldsymbol{r})\right]}} \exp \left(-\frac{U(\boldsymbol{r})+p^{2} / 2 m}{k_{\mathrm{B}} T}\right) 4 \pi p^{2} \mathrm{~d} p \\
& =\mathrm{P}\left[\frac{3}{2}, \kappa(\boldsymbol{r})\right] n_{0} \exp \left(-\frac{U(\boldsymbol{r})}{k_{\mathrm{B}} T}\right) .
\end{aligned}
$$

Similarly, the energy density is

$$
\begin{aligned}
e(\boldsymbol{r}) & =\frac{1}{(2 \pi \hbar)^{3}} \int \varepsilon(\boldsymbol{r}, \boldsymbol{p}) f(\boldsymbol{r}, \boldsymbol{p}) \mathrm{d}^{3} p \\
& =\left\{\frac{3}{2} k_{\mathrm{B}} T \mathrm{P}\left[\frac{5}{2}, \kappa(\boldsymbol{r})\right]+U(\boldsymbol{r}) \mathrm{P}\left[\frac{3}{2}, \kappa(\boldsymbol{r})\right]\right\} n_{0} \exp \left(-\frac{U(\boldsymbol{r})}{k_{\mathrm{B}} T}\right) .
\end{aligned}
$$




\section{Inelastic collisions}

Inelastic collisions occur with background gas atoms, between pairs of trapped atoms and between three trapped atoms. Corresponding loss rates are

$$
\begin{aligned}
& \dot{N}_{\mathrm{bgr}}=-\frac{1}{\tau} \int n(\boldsymbol{r}) \mathrm{d}^{3} r=-N / \tau, \\
& \dot{N}_{2 \mathrm{~b}}=-G \int n^{2}(\boldsymbol{r}) \mathrm{d}^{3} r \\
& \dot{N}_{3 \mathrm{~b}}=-L \int n^{3}(\boldsymbol{r}) \mathrm{d}^{3} r
\end{aligned}
$$

respectively; corresponding energy loss rates are

$$
\begin{aligned}
\dot{E}_{\mathrm{bgr}} & =-\frac{1}{\tau} \int e(\boldsymbol{r}) \mathrm{d}^{3} r=-E / \tau, \\
\dot{E}_{2 \mathrm{~b}} & =-G \int e(\boldsymbol{r}) n(\boldsymbol{r}) \mathrm{d}^{3} r \\
\dot{E}_{3 \mathrm{~b}} & =-L \int e(\boldsymbol{r}) n^{2}(\boldsymbol{r}) \mathrm{d}^{3} r
\end{aligned}
$$

respectively. A dot denotes a derivative with respect to time. The integrals have to be calculated numerically for every time step. The constant $\tau$ is the lifetime of the trap, determined experimentally. Fedichev et al. [12, 13] have calculated the other two constants for spin-polarized metastable helium. For $T<0.1 \mathrm{mK}, G$ is only dependent on magnetic field. The maximum value is $6 \times 10^{-13} \mathrm{~cm}^{3} / \mathrm{s}$ at $750 \mathrm{G}$, but as the contribution of two-body collisions is more significant towards the end of the sweep when the cloud is concentrated at the center, the value for $B \leq 10 \mathrm{G}$, which is $G=3 \times 10^{-14} \mathrm{~cm}^{3} / \mathrm{s}$, can be used [12]. The three-body loss-rate constant is given by $L=11.6 \hbar a^{4} / m$, with scattering length $a[13]$.

\section{Spilling}

When the trap shape or depth is changed, the eigenstates of the trapping potential with highest energies can become unbound. Atoms in these states are spilled from the trap. This process does not depend on collisions; when the potential is changed only by lowering the truncation energy $\varepsilon_{\mathrm{t}}$, spilling does not alter parameters $T$ and $n_{0}$. After instantaneous lowering of the trap depth from $\varepsilon_{\mathrm{t}}$ to $\varepsilon_{\mathrm{t}}^{\prime}$, the change in the number of atoms due to spilling is $\Delta N_{\theta}=-\int_{\varepsilon_{\mathrm{t}}^{\prime}}^{\varepsilon_{\mathrm{t}}} \rho(\varepsilon) f(\varepsilon) \mathrm{d} \varepsilon$ and the corresponding change in energy is $\Delta E_{\theta}=-\int_{\varepsilon_{\mathrm{t}}^{\prime}}^{\varepsilon_{\mathrm{t}}} \varepsilon \rho(\varepsilon) f(\varepsilon) \mathrm{d} \varepsilon$. With $n_{0} \Lambda^{3}=N / \zeta$ and Eq. (9), integration yields

$$
\begin{aligned}
& \Delta N_{\theta}=-N\left[1-\frac{3 \mathrm{P}\left(4, \eta^{\prime}\right)+2 \frac{U_{0}}{k_{\mathrm{B}} T} \mathrm{P}\left(3, \eta^{\prime}\right)}{3 \mathrm{P}(4, \eta)+2 \frac{U_{0}}{k_{\mathrm{B}} T} \mathrm{P}(3, \eta)}\right]=-N\left[1-\frac{\zeta\left(\eta^{\prime}\right)}{\zeta(\eta)}\right] \\
& \Delta E_{\theta}=-N k_{\mathrm{B}} T \frac{12 \mathrm{P}(5, \eta)+6 \frac{U_{0}}{k_{\mathrm{B}} T} \mathrm{P}(4, \eta)-12 \mathrm{P}\left(5, \eta^{\prime}\right)-6 \frac{U_{0}}{k_{\mathrm{B}} T} \mathrm{P}\left(4, \eta^{\prime}\right)}{3 \mathrm{P}(4, \eta)+2 \frac{U_{0}}{k_{\mathrm{B}} T} \mathrm{P}(3, \eta)}
\end{aligned}
$$

where $\eta^{\prime}=\varepsilon_{\mathrm{t}}^{\prime} / k_{\mathrm{B}} T \leq \eta$; the values of $N$ and $T$ are those before the step takes place.

\section{Evaporation}

After an elastic collision of two trapped atoms with energy $\varepsilon<\varepsilon_{\mathrm{t}}$, one atom may have an energy $\varepsilon>\varepsilon_{\mathrm{t}}$ and leave the trap. After this thermal escape, or evaporation, the average energy per atom has become smaller. The number and energy loss rates due to evaporation are

$$
\begin{aligned}
& \dot{N}_{\mathrm{ev}}=-\sqrt{\frac{8 k_{\mathrm{B}} T}{\pi m}} n_{0}^{2} \sigma \mathrm{e}^{-\eta} V_{\mathrm{ev}} \\
& \dot{E}_{\mathrm{ev}}=\dot{N}_{\mathrm{ev}}\left(\eta+\frac{W_{\mathrm{ev}}}{V_{\mathrm{ev}}}\right) k_{\mathrm{B}} T
\end{aligned}
$$


with effective volumes for evaporation

$$
\begin{aligned}
V_{\mathrm{ev}} & =\Lambda^{3} \zeta_{\infty}^{0}\left\{\left(1+\frac{2}{3} \frac{U_{0}}{k_{\mathrm{B}} T}\right)\left[\eta-\sum_{i=1}^{4} \mathrm{P}(i, \eta)\right]-\mathrm{P}(5, \eta)\right\}, \\
W_{\mathrm{ev}} & =\Lambda^{3} \zeta_{\infty}^{0}\left\{\left(1+\frac{2}{3} \frac{U_{0}}{k_{\mathrm{B}} T}\right)\left[\eta-\sum_{i=1}^{5} \mathrm{P}(i, \eta)\right]-\mathrm{P}(6, \eta)\right\}
\end{aligned}
$$

and elastic scattering cross section $\sigma$. The model has been developed for an energy-independent $\operatorname{cross}$ section $\sigma=8 \pi a^{2}$.

\section{Incorporation of an energy-dependent cross section}

The model as described in the previous section is restricted to the low-temperature limit, where $\sigma$ is energyindependent. In this section the theory will be adapted to include energy-dependence, although remaining in the $\mathrm{s}$-wave regime. In our experiment the temperature at the start of the rf sweep is about $1 \mathrm{mK}$, where the dependence of $\sigma$ on the relative velocity of the colliding atoms cannot be neglected. For the derivation of atom loss rate $\dot{N}_{\mathrm{ev}}$ and energy loss rate $\dot{E}_{\text {ev }}$ with an energy-dependent cross section $\sigma$, Luiten's derivation [14 is adapted. The elastic collision event rate $\Gamma_{\mathrm{c}}$, which is the number of collisions per second occurring in the whole cloud, is also derived. The average collision rate per atom is $2 \Gamma_{\mathrm{c}} / N$. This rate is not needed in the sweep simulation, but it is an interesting quantity, related to the atom loss rate $\dot{N}_{\text {ev }}$, which is the collision event rate under the condition that afterwards one of the atoms has enough energy to leave the trap.

\section{Collision event rate}

First the collision rate in the case of an (untruncated) Boltzmann velocity distribution is examined. The two-body elastic collision event rate is

$$
\Gamma_{\mathrm{c}}=\int \frac{1}{2} n(\boldsymbol{r})^{2}\left\langle\sigma v_{\mathrm{r}}\right\rangle \mathrm{d}^{3} r
$$

where rate coefficient $\left\langle\sigma v_{\mathrm{r}}\right\rangle$ is the product of cross section $\sigma$ and relative velocity $v_{\mathrm{r}}$ of the colliding pair of atoms, averaged over the thermal velocity distribution

$$
\frac{1}{2 \sqrt{\pi}}\left(\frac{m}{k_{\mathrm{B}} T}\right)^{3 / 2} v_{\mathrm{r}}^{2} \exp \left(-\frac{m v_{\mathrm{r}}^{2}}{4 k_{\mathrm{B}} T}\right) \mathrm{d} v_{\mathrm{r}} .
$$

Assuming the rate coefficient is not dependent on position due to the magnetic field, the collision event rate can be written as

$$
\Gamma_{\mathrm{c}}=\frac{N}{2} \frac{V_{2 \mathrm{e}}}{V_{\mathrm{e}}} n_{0}\left\langle\sigma v_{\mathrm{r}}\right\rangle
$$

and the average collision rate per atom as $\left(V_{2 \mathrm{e}} / V_{\mathrm{e}}\right) n_{0}\left\langle\sigma v_{\mathrm{r}}\right\rangle$, with

$$
\begin{aligned}
V_{\mathrm{e}} & =\int \frac{n(\boldsymbol{r})}{n_{0}} \mathrm{~d}^{3} r=N / n_{0}, \\
V_{2 \mathrm{e}} & =\int\left(\frac{n(\boldsymbol{r})}{n_{0}}\right)^{2} \mathrm{~d}^{3} r .
\end{aligned}
$$

For a Gaussian shaped density distribution $V_{2 \mathrm{e}} / V_{\mathrm{e}}=1 / \sqrt{8}$, for the density distribution in an IQ trap with $U_{0}=0$ (and infinite $\eta) V_{2 \mathrm{e}} / V_{\mathrm{e}}=1 / \sqrt{32}$. The collision rate at position $\boldsymbol{r}$ is $n(\boldsymbol{r})\left\langle\sigma v_{\mathrm{r}}\right\rangle$. Using the approximation $\sigma=8 \pi a^{2} /\left(k^{2} a^{2}+1\right)$ with thermal wave vector $k=m v_{\mathrm{r}} /(2 \hbar)$, we get

$$
\left\langle\sigma v_{\mathrm{r}}\right\rangle=8 \pi a^{2}\left\langle v_{\mathrm{r}}\right\rangle\left[\xi_{\mathrm{c}}-\xi_{\mathrm{c}}^{2} \mathrm{e}^{\xi_{\mathrm{c}}} \Gamma\left(0, \xi_{\mathrm{c}}\right)\right],
$$

with shorthand notation $\xi_{\mathrm{c}}=\hbar^{2} /\left(a^{2} m k_{\mathrm{B}} T\right)$ and average relative velocity $\left\langle v_{\mathrm{r}}\right\rangle=4 \sqrt{k_{\mathrm{B}} T /(\pi m)}$. Figure 1 shows the quantity in square brackets and the temperature dependence of the rate coefficient in the case of metastable helium.

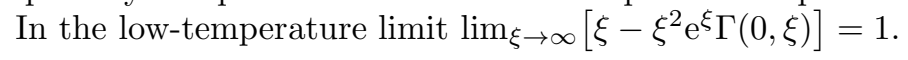



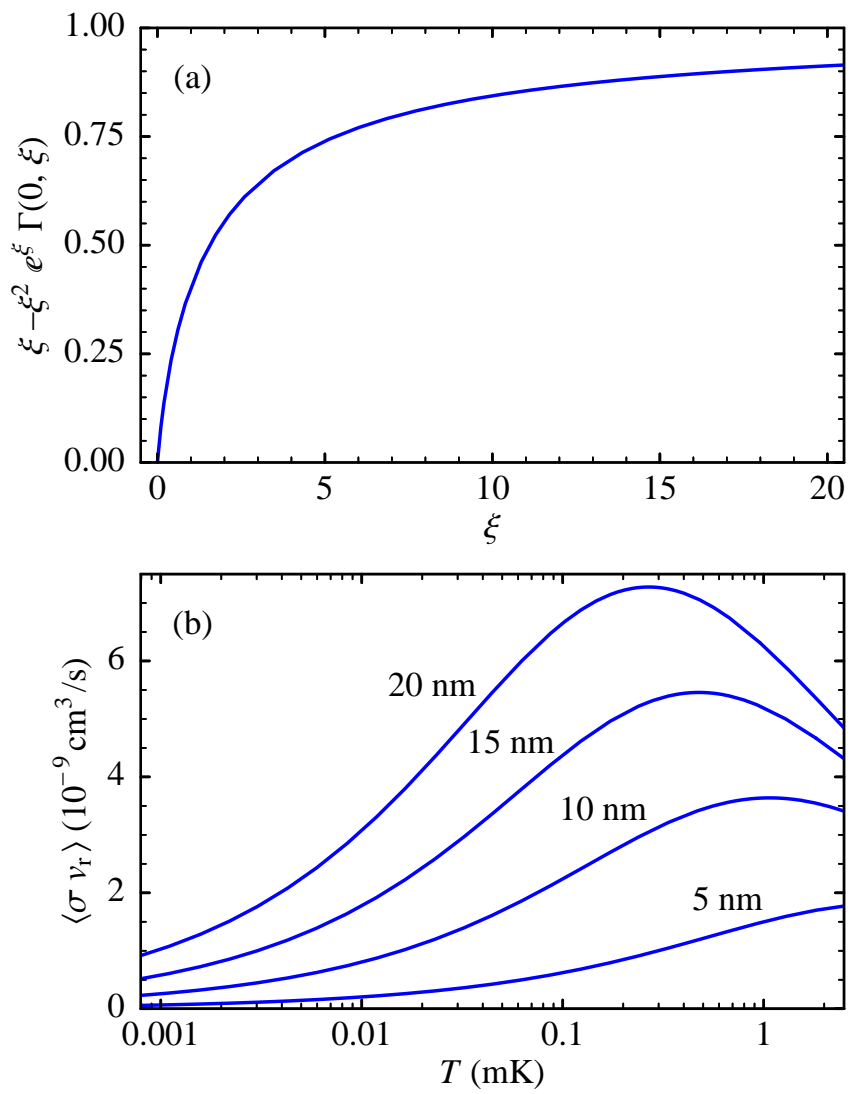

FIG. 1: (a) Factor $\left\langle\sigma v_{\mathrm{r}}\right\rangle /\left(8 \pi a^{2}\left\langle v_{\mathrm{r}}\right\rangle\right)$ as a function of $\xi$, and (b) the rate coefficient as a function of temperature for metastable helium assuming four different values for the scattering length $a$.

For a truncated Boltzmann distribution the collision event rate can be written as

$$
\Gamma_{\mathrm{c}}=\frac{1}{2 m(2 \pi \hbar)^{6}} \iiint \sigma\left(\left|\boldsymbol{p}_{2}-\boldsymbol{p}_{1}\right|\right)\left|\boldsymbol{p}_{2}-\boldsymbol{p}_{1}\right| f\left(\boldsymbol{r}, \boldsymbol{p}_{1}\right) f\left(\boldsymbol{r}, \boldsymbol{p}_{2}\right) \mathrm{d}^{3} p_{1} \mathrm{~d}^{3} p_{2} \mathrm{~d}^{3} r .
$$

The integration is over all $\left(\boldsymbol{r}, \boldsymbol{p}_{i}\right)$ with $U(\boldsymbol{r})+p_{i}^{2} / 2 m \leq \varepsilon_{\mathrm{t}}$ for both $i=1$ and $i=2$. A more convenient coordinate system uses average momentum $\boldsymbol{P}=\left(\boldsymbol{p}_{1}+\boldsymbol{p}_{2}\right) / 2$ and relative momentum $\boldsymbol{q}=\boldsymbol{p}_{2}-\boldsymbol{p}_{1}$, with angle $\theta$ between $\boldsymbol{P}$ and $\boldsymbol{q}$ and azimuthal angle $\varphi$. With Jacobian $\mathrm{d}^{3} p_{1} \mathrm{~d}^{3} p_{2}=\mathrm{d}^{3} P \mathrm{~d}^{3} q$ we get

$$
\Gamma_{\mathrm{c}}=\frac{n_{0}^{2} \Lambda^{6}}{2 m(2 \pi \hbar)^{6}} \int \mathrm{e}^{-2 U / k_{\mathrm{B}} T} \int \sigma(q) q \mathrm{e}^{-q^{2} / 4 m k_{\mathrm{B}} T} \int \mathrm{e}^{-P^{2} / m k_{\mathrm{B}} T} \mathrm{~d}^{3} P \mathrm{~d}^{3} q \mathrm{~d}^{3} r
$$

where the integration range is now given by

$$
P^{2}+\frac{q^{2}}{4}+P q|\cos \theta| \leq 2 m\left[\varepsilon_{\mathrm{t}}-U(\boldsymbol{r})\right] .
$$

To eliminate the angle dependence, the integrand is multiplied by the fraction $F$ of colliding pairs at position $\boldsymbol{r}$ for fixed $P$ and $q$ that are part of the truncated Boltzmann distribution:

$$
F=\frac{1}{4 \pi} \int_{0}^{2 \pi} \int \sin \theta \mathrm{d} \theta \mathrm{d} \varphi,
$$

where the integration over $\theta$ is restricted by condition (37). The result is

$$
F=\frac{Q^{2}-P^{2}-q^{2} / 4}{P q},
$$


with $Q=\sqrt{2 m\left[\varepsilon_{\mathrm{t}}-U(\boldsymbol{r})\right]}$ the maximum momentum an atom can have at position $\boldsymbol{r}$ without leaving the trap. This fraction has to be restricted to the physical range between 0 and 1. For $P>\left(Q^{2}-q^{2} / 4\right)^{1 / 2}$ at least one of the colliding atoms would have more momentum than is possible at position $r$ and the fraction is zero. For $0<P<Q-q / 2$ all angles $\theta$ are possible and the fraction is one. The integration over $\boldsymbol{P}$ is therefore divided into two parts:

$$
\begin{aligned}
\Gamma_{\mathrm{c}}=\frac{n_{0}^{2} \Lambda^{6}}{2 m(2 \pi \hbar)^{6}} \int \mathrm{e}^{-2 U / k_{\mathrm{B}} T} \int_{0}^{2 Q} \sigma(q) q \mathrm{e}^{-q^{2} / 4 m k_{\mathrm{B}} T} & \\
& \left(\int_{0}^{Q-q / 2} \mathrm{e}^{-P^{2} / m k_{\mathrm{B}} T} \mathrm{~d}^{3} P+\int_{Q-q / 2}^{\sqrt{Q^{2}-q^{2} / 4}} F \mathrm{e}^{-P^{2} / m k_{\mathrm{B}} T} \mathrm{~d}^{3} P\right) \mathrm{d}^{3} q \mathrm{~d}^{3} r .
\end{aligned}
$$

For an IQ trap [Eq. (10], the integration over position can be made one-dimensional 2] using

$$
\int \mathcal{F}[U(\boldsymbol{r})] \mathrm{d}^{3} r=\frac{4 \pi}{\alpha^{2} \sqrt{\beta}} \int_{0}^{\varepsilon_{\mathrm{t}}} \sqrt{U}\left(U+U_{0}\right) \mathcal{F}(U) \mathrm{d} U .
$$

Introducing scaled variables $y=q / \sqrt{m k_{\mathrm{B}} T}$ and $\kappa=\left(\varepsilon_{\mathrm{t}}-U\right) / k_{\mathrm{B}} T$, and scaled constant $\eta=\varepsilon_{\mathrm{t}} / k_{\mathrm{B}} T$ the collision event rate after integration over $\boldsymbol{P}$ becomes

$$
\begin{aligned}
& \Gamma_{\mathrm{c}}=\frac{n_{0}^{2} \mathrm{e}^{-2 \eta}}{\alpha^{2} \sqrt{\beta m}}\left(k_{\mathrm{B}} T\right)^{3} \int_{0}^{\eta} \int_{0}^{\sqrt{8 \kappa}} \sigma(y) \sqrt{\eta-\kappa}\left(\eta-\kappa+\frac{U_{0}}{k_{\mathrm{B}} T}\right) y^{2} \\
& {\left[2-2 \exp \left(\sqrt{2 \kappa} y-\frac{1}{2} y^{2}\right)+\sqrt{\pi} y \exp \left(2 \kappa-\frac{1}{4} y^{2}\right) \operatorname{erf}\left(\sqrt{2 \kappa}-\frac{1}{2} y\right)\right] \mathrm{d} y \mathrm{~d} \kappa . }
\end{aligned}
$$

For an energy-dependent cross section $\sigma$ with $\hbar k=q / 2$ this equation has to be integrated numerically. For a constant cross section, after integration over $y$, the expression for $\Gamma_{\mathrm{c}}$ contains terms

$$
\int_{0}^{\eta}(\eta-\kappa)^{x} \mathrm{e}^{l \kappa} \operatorname{erfc} \sqrt{l \kappa} \mathrm{d} \kappa=\Gamma(x+1) \mathrm{e}^{l \eta}\left[\mathrm{P}(x+1, l \eta)-\mathrm{P}\left(x+\frac{3}{2}, l \eta\right)\right] / l^{x+1}
$$

and

$$
\begin{aligned}
\int_{0}^{\eta}(\eta-\kappa)^{x} \kappa \mathrm{e}^{\kappa} \operatorname{erfc} \sqrt{\kappa} \mathrm{d} \kappa=\Gamma(x+1) \mathrm{e}^{\eta}\{(\eta+1) & {\left[\mathrm{P}(x+2, \eta)-\mathrm{P}\left(x+\frac{7}{2}, \eta\right)\right] } \\
& \left.-\frac{3}{2} \mathrm{P}\left(x+\frac{5}{2}, \eta\right)-(x+2) \mathrm{P}(x+3, \eta)+\left(x+\frac{7}{2}\right) \mathrm{P}\left(x+\frac{9}{2}, \eta\right)\right\},
\end{aligned}
$$

with Euler gamma function $\Gamma(z)=\Gamma(z, 0)$ and complementary error function $\operatorname{erfc}(z)=1-\operatorname{erf}(z)$, which has the property [15]

$$
\mathrm{e}^{\kappa} \operatorname{erfc} \sqrt{\kappa}=\frac{\sqrt{\kappa}}{\pi} \int_{0}^{\infty} \frac{\mathrm{e}^{-t}}{\sqrt{t}(t+\kappa)} \mathrm{d} t
$$

The collision event rate for constant $\sigma$ becomes

$$
\begin{aligned}
\Gamma_{\mathrm{c}}=\frac{1}{2 \sqrt{2}} n_{0}^{2} \sigma \sqrt{\frac{k_{\mathrm{B}} T}{\pi m}} \Lambda^{3} \zeta_{\infty}\left\{1+\frac{4}{3} \frac{U_{0}}{k_{\mathrm{B}} T}-8 \mathrm{e}^{-\eta}\left(\eta-1+\frac{2}{3} \frac{U_{0}}{k_{\mathrm{B}} T} \eta\right)\right. \\
\left.+\frac{1}{3} \mathrm{e}^{-2 \eta}\left[\eta^{4}+4 \eta^{3}+6 \eta^{2}-6 \eta-27+\frac{4}{3} \frac{U_{0}}{k_{\mathrm{B}} T}\left(2 \eta^{3}+6 \eta^{2}+6 \eta-3\right)\right]\right\} .
\end{aligned}
$$

When $\sigma$ is energy-dependent, $\Gamma_{\mathrm{c}}$ needs to be calculated numerically. However, in the limit of large $\eta$ the velocity distribution becomes thermal and Eq. (42) is expected to produce the same result as Eq. (31). For common values of $\eta$ and a cross section given by $\sigma=8 \pi a^{2} /\left(k^{2} a^{2}+1\right)$, Eqs. (31) and (34) can be used as an approximation: comparing exact numerical calculations of $I_{\mathrm{c}} /\left(\frac{N}{2} \frac{V_{2 \mathrm{e}}}{V_{\mathrm{e}}} n_{0}\right)$ with the rate coefficient as given by Eq. (34), at $\eta=10$ deviations are smaller than $0.1 \%$ and at $\eta=5$ smaller than $6 \%$ (for $m<100 \mathrm{u},|a|<100 \mathrm{~nm}, U_{0} / \mu_{\mathrm{B}}<100 \mathrm{G}, T<5 \mathrm{mK}$ ).

\section{Evaporation rates}

The collision event rate $\Gamma_{\mathrm{c}}$ is given by Eq. (36), with constraints to the integration range incorporated by a factor $F$ in the integrand. The atom loss rate $\dot{N}_{\mathrm{ev}}$ is given by the same equation, but with an extra factor for the fraction 
of collisions for which afterwards one of the atoms has enough energy to leave the trap. Since $P$ and $q$ are the same before and after collisions and all scattering angles $\theta^{\prime}$ have equal probability in the s-wave regime, this factor is $(1-F)$. In the case of the collision event rate, the integration over $P$ had to be divided into two parts [see Eq. (40)]. Here only one part is left, as the integrand including $F(1-F)$ with $F=1$ is zero. The evaporation rate becomes

$$
\begin{gathered}
\dot{N}_{\mathrm{ev}}=-\frac{4 \pi n_{0}^{2} \Lambda^{6}}{\alpha^{2} \sqrt{\beta} 2 m(2 \pi \hbar)^{6}} \int_{0}^{\varepsilon_{\mathrm{t}}} \sqrt{U}\left(U+U_{0}\right) \mathrm{e}^{-2 U / k_{\mathrm{B}} T} \int_{0}^{2 Q} \sigma(q) 4 \pi q^{3} \mathrm{e}^{-q^{2} / 4 m k_{\mathrm{B}} T} \\
\int_{Q-q / 2}^{\sqrt{Q^{2}-q^{2} / 4}} F(1-F) 4 \pi P^{2} \mathrm{e}^{-P^{2} / m k_{\mathrm{B}} T} \mathrm{~d} P \mathrm{~d} q \mathrm{~d} U,
\end{gathered}
$$

and after integration over $P$ :

$$
\begin{aligned}
& \dot{N}_{\mathrm{ev}}=-\frac{n_{0}^{2} \mathrm{e}^{-2 \eta}}{8 \alpha^{2} \sqrt{\beta m}}\left(k_{\mathrm{B}} T\right)^{3} \int_{0}^{\eta} \int_{0}^{\sqrt{8 \kappa}} \sigma(y) \sqrt{\eta-\kappa}\left(\eta-\kappa+\frac{U_{0}}{k_{\mathrm{B}} T}\right) y \\
&\left\{-2\left[y\left(2+y^{2}-8 \kappa\right)+2 \sqrt{2 \kappa}\left(6+y^{2}-8 \kappa\right)\right] \exp \left(\sqrt{2 \kappa} y-\frac{1}{2} y^{2}\right)\right. \\
&+2\left[8 y+\sqrt{8 \kappa-y^{2}}\left(6+y^{2}-8 \kappa\right)\right]-\sqrt{\pi}\left[12+4 y^{2}+y^{4}-16\left(2+y^{2}\right) \kappa+64 \kappa^{2}\right] \\
&\left.\quad \exp \left(2 \kappa-\frac{1}{4} y^{2}\right)\left[\operatorname{erf}\left(\sqrt{2 \kappa-\frac{1}{4} y^{2}}\right)-\operatorname{erf}\left(\sqrt{2 \kappa}-\frac{1}{2} y\right)\right]\right\} \mathrm{d} y \mathrm{~d} \kappa .
\end{aligned}
$$

For the energy loss rate $\dot{E}_{\text {ev }}$ there is an extra factor in the integrand for the energy of the lost atom,

$$
\varepsilon_{4}=U+\frac{1}{2 m}\left(P^{2}+\frac{q^{2}}{4}+P q\left|\cos \theta^{\prime}\right|\right) .
$$

This means $\dot{E}_{\text {ev }}$ is given by Eq. (47) with the factor $(1-F)$ replaced by

$$
G=\frac{1}{4 \pi} \int_{0}^{2 \pi} \int \varepsilon_{4} \sin \theta^{\prime} \mathrm{d} \theta^{\prime} \mathrm{d} \varphi^{\prime}
$$

where the integration range is given by $\varepsilon_{4}>\varepsilon_{\mathrm{t}}$. After integration over $\theta^{\prime}$ and $\varphi^{\prime}$ we get

$$
G=(1-F)\left[\varepsilon_{\mathrm{t}}+\frac{(2 P+q)^{2}-4 Q^{2}}{16 m}\right] .
$$

The energy loss rate after integration over $P$ becomes

$$
\begin{gathered}
\dot{E}_{\mathrm{ev}}=\varepsilon_{\mathrm{t}} \dot{N}_{\mathrm{ev}}-\frac{n_{0}^{2} \mathrm{e}^{-2 \eta}}{128 \alpha^{2} \sqrt{\beta m}}\left(k_{\mathrm{B}} T\right)^{4} \int_{0}^{\eta} \int_{0}^{\sqrt{8 \kappa}} \sigma(y) \sqrt{\eta-\kappa}\left(\eta-\kappa+\frac{U_{0}}{k_{\mathrm{B}} T}\right) y \\
\left\{256 y+2 \sqrt{8 \kappa-y^{2}}\left[60+y^{4}-16 y^{2}(\kappa-2)+64 \kappa(\kappa-1)\right]\right. \\
\quad-2\left\{y^{5}+2 y^{4} \sqrt{2 \kappa}-4 y^{3}(4 \kappa-3)-16 y^{2} \sqrt{2 \kappa}(2 \kappa-1)\right. \\
+y[68+32 \kappa(2 \kappa-3)]+8 \sqrt{2 \kappa}[15+16 \kappa(\kappa-1)]\} \exp \left(\sqrt{2 \kappa} y-\frac{1}{2} y^{2}\right) \\
-\sqrt{\pi}\left(y^{6}-2 y^{4}(12 \kappa-7)+4 y^{2}[21+8 \kappa(6 \kappa-5)]+8\{15-4 \kappa[9+4 \kappa(4 \kappa-3)]\}\right) \\
\left.\exp \left(2 \kappa-\frac{1}{4} y^{2}\right)\left[\operatorname{erf}\left(\sqrt{2 \kappa-\frac{1}{4} y^{2}}\right)-\operatorname{erf}\left(\sqrt{2 \kappa}-\frac{1}{2} y\right)\right]\right\} \mathrm{d} y \mathrm{~d} \kappa
\end{gathered}
$$

When $\sigma$ is a constant, Eqs. (48) and (52) reduce after integration to Eqs. (26) and (27), respectively. When $\sigma$ is energy-dependent, the expressions for $\dot{N}_{\mathrm{ev}}$ and $\dot{E}_{\mathrm{ev}}$ have to be integrated numerically. An effective cross section $\sigma_{\text {eff }}$ can be introduced, which will give the correct value of $\dot{N}_{\text {ev }}$ if $\sigma=\sigma_{\text {eff }}$ in Eq. (26). In the case that the cross section is given by $\sigma=8 \pi a^{2} /\left(k^{2} a^{2}+1\right)$, the effective cross section can be approximated by a variation of Eq. (34):

$$
\sigma_{\mathrm{eff}} \approx 8 \pi a^{2}\left[\xi_{\mathrm{ev}}-\xi_{\mathrm{ev}}^{2} \mathrm{e}^{\xi_{\mathrm{ev}}} \Gamma\left(0, \xi_{\mathrm{ev}}\right)\right]
$$




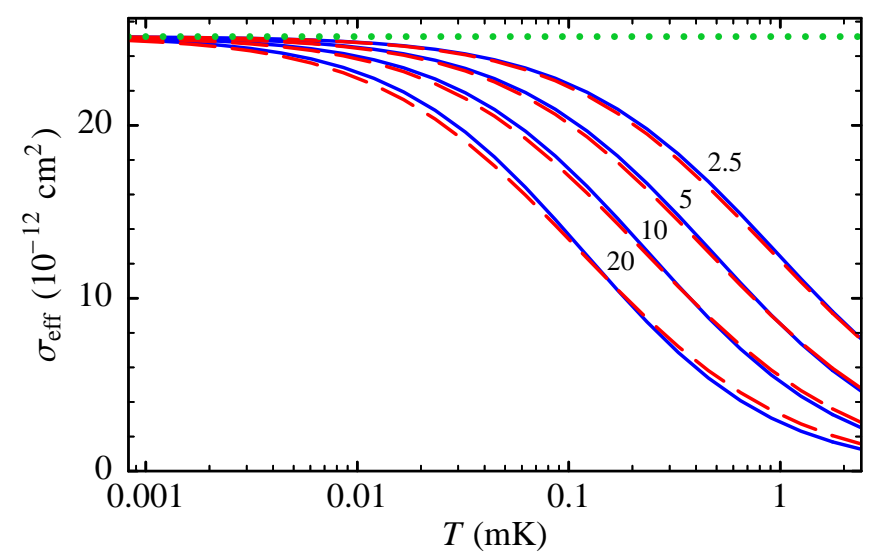

FIG. 2: Effective cross section as a function of temperature for $m=4 \mathrm{u}, a=10 \mathrm{~nm}, B_{0}=0.46 \mathrm{G}$ and four values of $\eta$ (solid curves), together with the approximation (dashed curves). The dotted line is the low-temperature limit.

where

$$
\xi_{\mathrm{ev}}=\frac{3}{\eta} \frac{\hbar^{2}}{m k_{\mathrm{B}} T a^{2}}=\frac{3 \hbar^{2}}{\varepsilon_{\mathrm{t}} m a^{2}} .
$$

In this approximation, found by trial and error, the effective cross section is only dependent on temperature via the trap depth. Figure [2] shows $\sigma_{\text {eff }}$ and its approximation as a function of temperature in our situation for $a=10 \mathrm{~nm}$ and several values of $\eta$. Apparently $\sigma_{\text {eff }}(T)$ does not approach an asymptote at high $\eta$. In general $(m<100 \mathrm{u}$, $\left.|a|<100 \mathrm{~nm}, U_{0} / \mu_{\mathrm{B}}<100 \mathrm{G}, T<5 \mathrm{mK}, 2<\eta<25\right)$ the absolute difference between $\sigma_{\text {eff }}$ and its approximation is less than $3 \%$ of the low-temperature limit $8 \pi a^{2}$, shown as a dotted line in Fig. 2 The energy loss rate can still be calculated with Eq. (27), even though the effective volumes are not correct: the relative error in $\dot{E}_{\text {ev }} / \dot{N}_{\text {ev }}$ is less than $2 \%$.

\section{EXPERIMENT}

We have performed evaporative cooling experiments in a setup which is described in detail by Herschbach et al. []. In short, an atomic beam from a DC discharge source of metastable helium is collimated, deflected and slowed in a traditional Zeeman slower. The atoms are trapped in a magneto-optical trap (MOT), cooled in optical molasses, spin-polarized by optical pumping, again trapped and finally compressed in a cloverleaf magnetic trap, an example of an IQ trap. The magnetic field configuration is determined by $\alpha /\left(2 \mu_{\mathrm{B}}\right)=69 \mathrm{G} / \mathrm{cm}, \beta /\left(2 \mu_{\mathrm{B}}\right)=13.9 \mathrm{G} / \mathrm{cm}^{2}$, $B_{0}=0.5 \mathrm{G}$, and the trap depth is $47 \mathrm{G}$ (corresponding to $6.4 \mathrm{mK}$ ). The harmonic part is characterized by trap frequencies $\omega_{\rho} /(2 \pi)=853 \mathrm{~Hz}$ and $\omega_{z} /(2 \pi)=44 \mathrm{~Hz}$. After compression a cloud of $\sim 10^{9}$ atoms at a temperature of $\sim 1 \mathrm{mK}$ and a central density of $\sim 10^{10} \mathrm{~cm}^{-3}$ is obtained. Temperature $T$ and a relative measure of the number of atoms are determined by time-of-flight (TOF) measurements with a microchannel plate (MCP) detector positioned $18 \mathrm{~cm}$ from the trap center, whereas calibration of the number of atoms $N$ is performed by absorption imaging in the MOT. As the radial confinement is much stronger than the axial confinement, the cloud is elongated: the theoretical density while the atoms are still in the trap has a FWHM of $0.2 \mathrm{~cm}$ horizontally and $1.4 \mathrm{~cm}$ vertically. A contour plot of the density, integrated in one horizontal dimension, is shown in Fig. 3(a). Absorption images are taken $1 \mathrm{~ms}$ after the trap has been switched off. To simulate the ballistic expansion during this time, the theoretical density distribution is convolved numerically with a Maxwell-Boltzmann velocity distribution before the integration in one dimension, using a temperature of $1.2 \mathrm{mK}$ determined from a TOF measurement [Fig. [3(b)]. No fitting is required and the shape agrees well with the experimental column density [Fig. [3 (c)]. In contrast, a Gaussian fit to the experimental column density deviates significantly [Fig. 3(d)]. At lower temperatures the cloud becomes smaller and a larger part is confined to the harmonic region of the trap. Therefore the density distribution approaches a Gaussian shape during evaporative cooling. However, only below $17 \mu \mathrm{K}$ will the r.m.s. radii calculated with a harmonic approximation of the trap deviate less than $10 \%$ from the exact solution.

For evaporative cooling, the rf frequency starts at $120 \mathrm{MHz}$ and decreases exponentially with a time constant of $5 \mathrm{~s}$, close to the optimum found by maximizing the elastic collision rate per atom after a sweep to $5 \mathrm{MHz}$. The progress of the temperature and number of atoms is followed both in absorption imaging and with the MCP detector. A series 


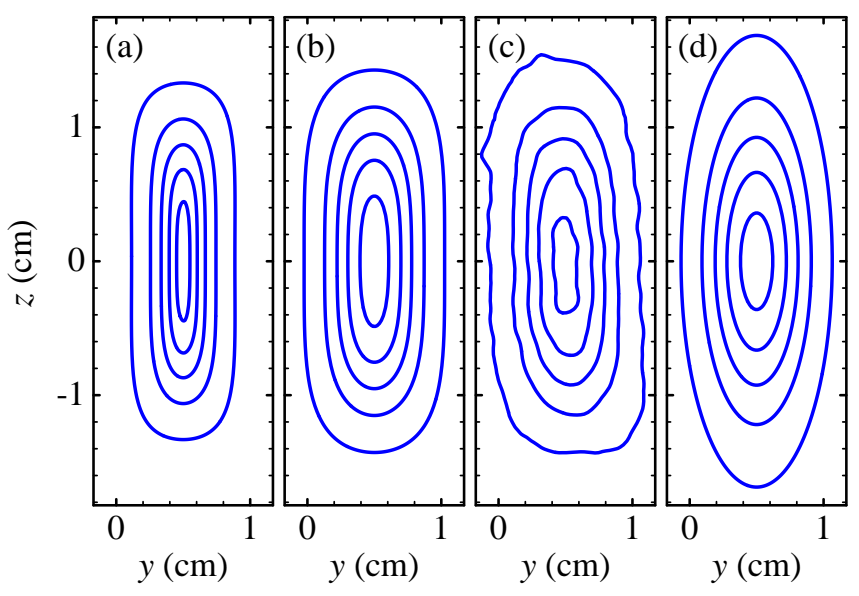

FIG. 3: The density distribution integrated in the $x$-direction. From left to right: the theoretical distribution (a) in the cloverleaf trap and (b) after $1 \mathrm{~ms}$ expansion, (c) the density from the experimental absorption image (the negative logarithm of the transmittance), and (d) a Gaussian fit to the experimental density distribution. Contours at $10 \%, 30 \%, 50 \%, 70 \%$ and $90 \%$ of the maximum integrated density.

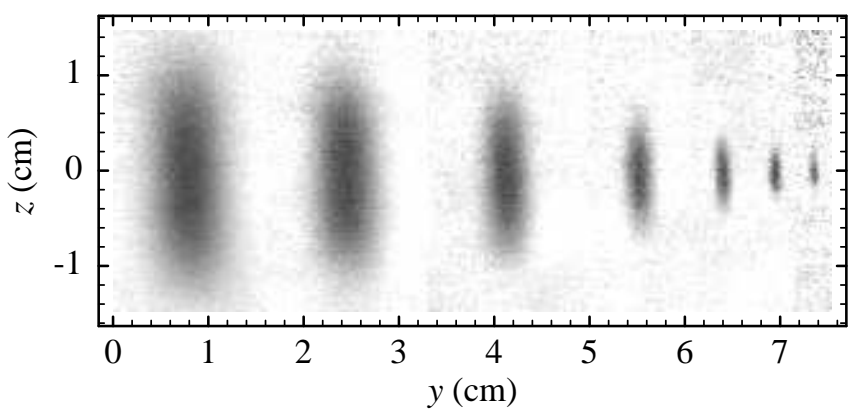

FIG. 4: Collection of absorption images taken $1 \mathrm{~ms}$ after the cloverleaf trap is switched off, at various stages during an rf sweep. From left to right: just before rf is turned on, at $100 \mathrm{MHz}, 65 \mathrm{MHz}, 35 \mathrm{MHz}, 15 \mathrm{MHz}, 10 \mathrm{MHz}$, and $5 \mathrm{MHz}$.

of images taken after $1 \mathrm{~ms}$ expansion is shown in Fig. 4 To emphasize the change in cloud size, maximum absorption within each image is rendered black. The central absorption varies from $62 \%$ in the leftmost image to $14 \%$ in the rightmost image. The smallest cloud contains $9(4) \times 10^{6}$ atoms at a temperature of $23(3) \mu \mathrm{K}$. Above $0.5 \mathrm{mK}$ the radii are larger than expected, especially in the horizontal plane, with a density that is half of the predicted value. This may be explained by a time lag between a change of trap depth and getting closer to steady state at a smaller cloud size: the sweep has to be executed rather fast, due to a limited trap lifetime of $12.5 \mathrm{~s}$. For theoretical calculations the actual size during the sweep is required. A first order correction of the modeled density distribution is to multiply trap parameters $\alpha$ and $\beta$ in Eq. (1) with factors that are kept constant during the sweep. These are $f_{\alpha}=0.74(9)$ and $f_{\beta}=0.89(5)$, respectively, where the uncertainty includes the variation in the measured radii and the temperature uncertainty.

Figure 5 shows the temperature and number of atoms as a function of time since the start of the rf sweep. Each point is the result of a TOF measurement after the sweep is interrupted by turning off the rf power and the trap at the same time. Error bars are given as a gray band, including a $20 \%$ standard deviation in the calibration of the number of atoms according to the MCP and including the uncertainty in the temperature due to magnetic field gradients between the trap and the MCP [16]. The indirect effect of the temperature error on the number of atoms is also taken into account. The line is a theoretical calculation, assuming the scattering length is $10 \mathrm{~nm}$. The trap lifetime is measured to be $12.5 \mathrm{~s}$, the measured heating of $0.7 \mu \mathrm{K} / \mathrm{s}$ is neglected, and the trap minimum is 0.5 G. Correction factors $f_{\alpha}$ and $f_{\beta}$ are included, so $\alpha /\left(2 \mu_{\mathrm{B}}\right)=51 \mathrm{G} / \mathrm{cm}$ and $\beta /\left(2 \mu_{\mathrm{B}}\right)=12.4 \mathrm{G} / \mathrm{cm}^{2}$. Due to an unexpected feature of the rf generator, below $7 \mathrm{MHz}$ the rf power drops, dwindling to zero below $4 \mathrm{MHz}$. This means the rf is effectively turned off after $17 \mathrm{~s}$, the temperature stays constant and the number of atoms goes down only due to background collisions.

The five loss rates used in the model are plotted as a function of time in Fig. [6 for the theoretical sweep with $a=10 \mathrm{~nm}$. The contribution of two- and three-body collisions can be neglected up to the moment BEC is reached, 

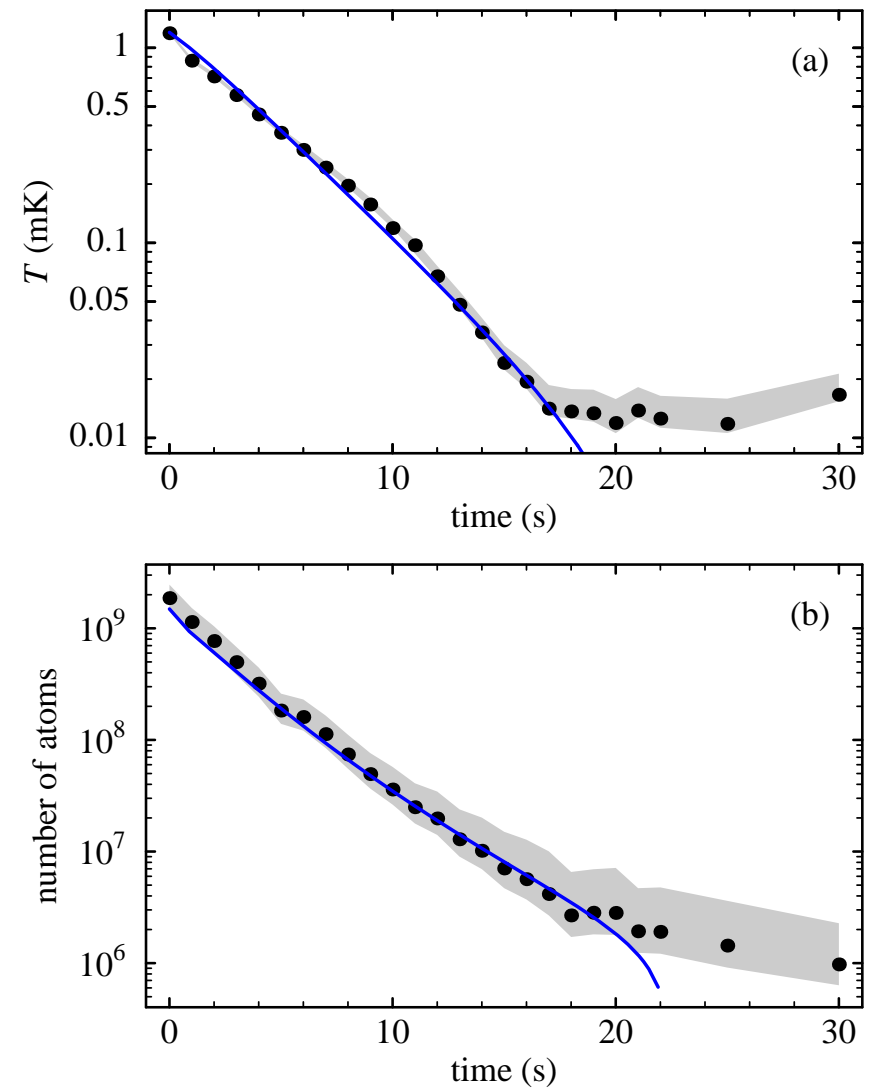

FIG. 5: (a) Temperature and (b) number of atoms as a function of rf time during a sweep. The gray bands show the possible values due to uncertainty in the detector calibration and in the temperature. The curve is a simulation with $a=10 \mathrm{~nm}$.

theoretically after $22 \mathrm{~s}$. At the start of the sweep the dominant loss process is spilling, because the frequency is decreased relatively fast. If the sweep would take longer, spilling losses would be reduced in favor of evaporation losses, but this would only be advantageous if the trap lifetime were larger. Figure 6 (b) shows that an exponentially decreasing rf frequency goes unnecessarily fast towards the end: the spilling contribution increases in the last seconds.

To find the scattering length best describing the data, one can simulate sweeps for several values of $a$ starting from the temperature and number of atoms in the beginning. However, deviations early on influence the behavior of the curve at later times. For instance, turning on the rf is not well modeled, as the experimental temperature after $1 \mathrm{~s}$ is lower than expected [see Fig. 国(a)]. In addition, the temperature as a function of time exhibits a slope change after $11 \mathrm{~s}$, which is not seen in simulations with any value of the scattering length. Therefore, small sweeps are simulated for each interval between the data points. Starting from the experimental situation at one point with a constant $\sigma$ in Eq. (26), the temperature at the end of the sweep is calculated. The values of $\sigma$ for which the end temperature according to the model corresponds to the experimental temperature are given in Fig. 7 (a). The curve is the effective cross section according to Eq. (53) for $a=10 \mathrm{~nm}$. The gray band indicates the error due to the uncertainty in the MCP calibration and the temperature (as in Fig. 5), as well as the uncertainty in the correction factor for the theoretical cloud size. The scattering length determined with the experimental effective cross section and Eq. (53) is given in Fig. 7(b). The point in the first interval is dropped, because the effective cross section was unusually high. This in turn is caused by the unexpectedly large drop in temperature after turning on the rf power, which makes evaporation look very effective. In the last part of the sweep the uncertainty in the scattering length becomes large due to the increasing uncertainty in the temperature determination. However, the possible error in $a$ in the first half of the sweep may be larger than given, because the evaporation process seems not to behave entirely according to the model. Also, at the start we have to rely more on the model anyway, as the scattering length is determined only from evaporation, which then constitutes just a quarter of the loss rate [Fig. [6 (b)]. Taking this into account a scattering length $a=10(5) \mathrm{nm}$ can be considered as consistent with the experimental data. 

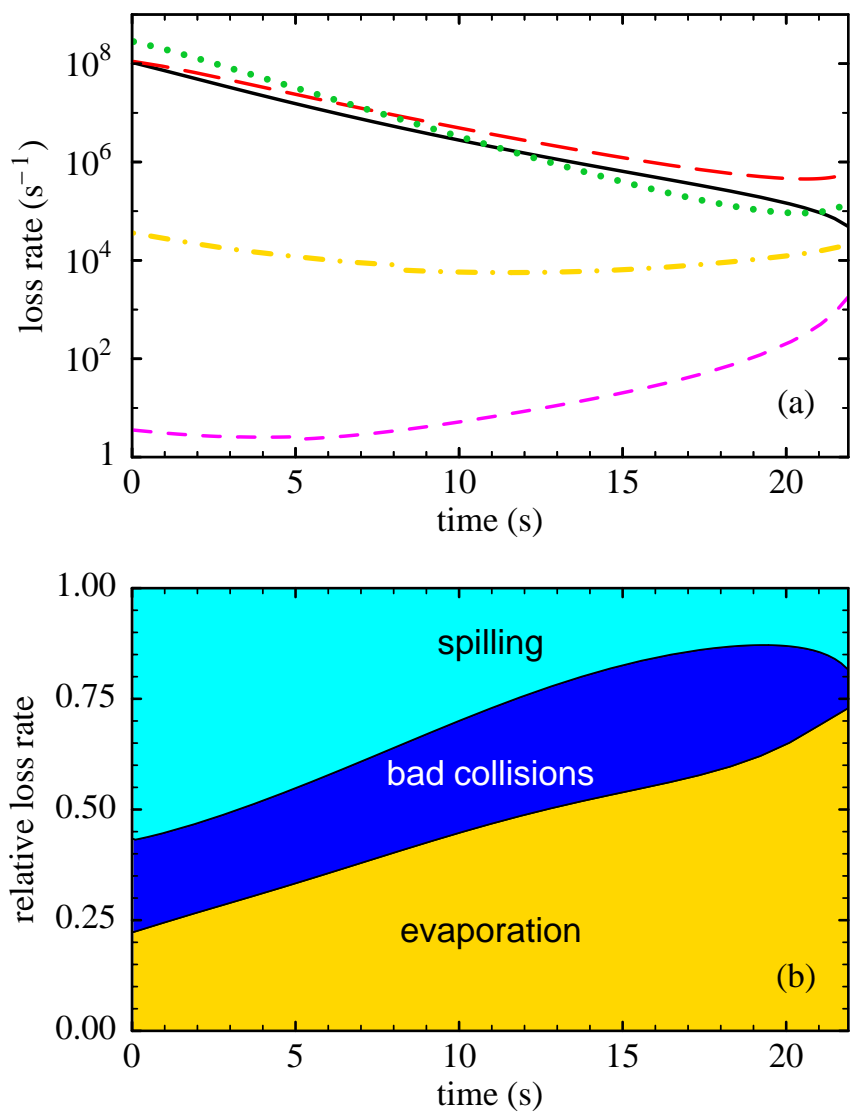

FIG. 6: (a) Absolute and (b) relative loss rates due to background collisions $\dot{N}_{\text {bgr }}$ (solid curve), evaporation $\dot{N}_{\text {ev }}$ (long-dashed curve), spilling $\dot{N}_{\theta}$ (dotted curve), two-body collisions $\dot{N}_{2 \mathrm{~b}}$ (dash-dotted curve) and three-body collisions $\dot{N}_{3 \mathrm{~b}}$ (small-dashed curve), as a function of sweep time. In (b) the three loss rates due to bad inelastic collisions are summed. This is according to a simulation with $a=10 \mathrm{~nm}$.

\section{SUMMARY AND CONCLUSIONS}

To summarize, we have developed a model that allows simulation of evaporative cooling experiments in situations where the elastic scattering cross section depends on collision energy. The model is used to simulate the number of atoms and temperature in an rf sweep for metastable helium. From a comparison with the experiment an s-wave scattering length $|a|=10(5) \mathrm{nm}$ is extracted. This value is a factor of two smaller but within the experimental accuracy of previous experiments; the BEC experiment of the Orsay group yields $a=+20(10) \mathrm{nm}$ [4, 17], the result of the ENS group is $a=+16(8) \mathrm{nm}[5$, , 18]. Later experiments of the ENS group are more consistent with a lower rather than a higher value [7]. The scattering length can also be determined theoretically, because the ${ }^{5} \Sigma_{\mathrm{g}}^{+}$potential in which spin-polarized atoms collide has been calculated. From the Stärck and Meyer potential [8] a scattering length $a=+8.3 \mathrm{~nm}$ is deduced. More recently, Gadéa, Leininger and Dickinson 10 calculated the short-range part of this potential more accurately and combining their potential with the accurately determined long-range potential of Yan and Babb [19] they determine $a=+15.4 \mathrm{~nm}$. However, the value of the scattering length is very sensitive to the way the short-range and long-range potential are connected. An improved method of combining the two potentials results in $a=+12.2_{-4.1}^{+0} \mathrm{~nm}[9]$. Our experimental result is in good agreement with this value. 

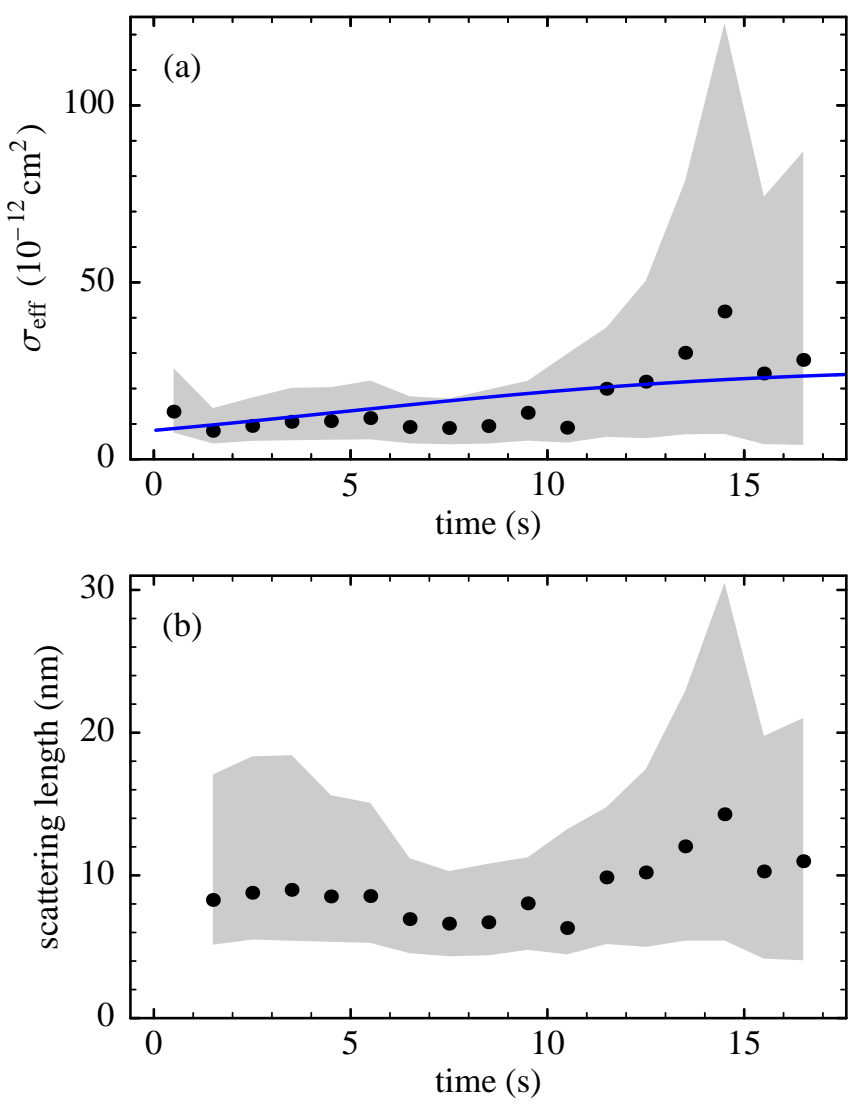

FIG. 7: (a) Effective cross section and (b) scattering length as a function of sweep time. The curve is a simulation with $a=10 \mathrm{~nm}$, the gray bands indicate the error due to uncertainty in the number of atoms, temperature and cloud size.

\section{Acknowledgments}

We gratefully acknowledge Norbert Herschbach for fruitful discussions and the Foundation for Fundamental Research on Matter (FOM) for financial support.

[1] W. Ketterle and N. J. van Druten, in Advances in Atomic, Molecular, and Optical Physics, edited by B. Bederson and H. Walther (Academic Press, San Diego, 1996), vol. 37, pp. 181-236.

[2] O. J. Luiten, M. W. Reynolds, and J. T. M. Walraven, Phys. Rev. A 53, 381 (1996).

[3] P. W. H. Pinkse, A. Mosk, M. Weidemüller, M. W. Reynolds, T. W. Hijmans, and J. T. M. Walraven, Phys. Rev. A 57, 4747 (1998).

[4] A. Robert, O. Sirjean, A. Browaeys, J. Poupard, S. Nowak, D. Boiron, C. I. Westbrook, and A. Aspect, Science 292, 461 (2001).

[5] F. Pereira dos Santos, J. Léonard, J. Wang, C. J. Barrelet, F. Perales, E. Rasel, C. S. Unnikrishnan, M. Leduc, and C. Cohen-Tannoudji, Phys. Rev. Lett. 86, 3459 (2001).

[6] N. Herschbach, P. J. J. Tol, A. S. Tychkov, W. Hogervorst, and W. Vassen, J. Opt. B: Quantum Semiclass. Opt. 5, S65 (2003).

[7] M. Leduc, J. Léonard, F. Pereira dos Santos, E. Jahier, S. Schwartz, and C. Cohen-Tannoudji, Acta Phys. Pol. B 33, 2213 (2002).

[8] J. Stärck and W. Meyer, Chem. Phys. Lett. 225, 229 (1994).

[9] A. S. Dickinson, F. X. Gadéa, and T. Leininger, J. Phys. B (to be published).

[10] F. X. Gadéa, T. Leininger, and A. S. Dickinson, J. Chem. Phys. 117, 7122 (2002).

[11] Incomplete gamma function $\mathrm{P}$ can be given in terms of incomplete gamma function $\Gamma$ as $\mathrm{P}(a, z)=1-\Gamma(a, z) / \Gamma(a, 0)=$ $\int_{0}^{z} t^{a-1} \mathrm{e}^{-t} \mathrm{~d} t / \int_{0}^{\infty} t^{a-1} \mathrm{e}^{-t} \mathrm{~d} t$

[12] P. O. Fedichev, M. W. Reynolds, U. M. Rahmanov, and G. V. Shlyapnikov, Phys. Rev. A 53, 1447 (1996), the loss rate 
constant for two-body inelastic collisions is $\alpha_{\text {in }}$ in Fig. 3.

[13] P. O. Fedichev, M. W. Reynolds, and G. V. Shlyapnikov, Phys. Rev. Lett. 77, 2921 (1996).

[14] O. J. Luiten, Ph.D. thesis, University of Amsterdam, 1993 (unpublished).

[15] M. Abramowitz and I. A. Stegun, eds., Handbook of Mathematical Functions with Formulas, Graphs, and Mathematical Tables, Applied Mathematics Series 55 (National Bureau of Standards, 1964).

[16] P. J. J. Tol, Ph.D. thesis, Vrije Universiteit Amsterdam, 2004 (unpublished).

[17] O. Sirjean, S. Seidelin, J. Viana Gomes, D. Boiron, C. I. Westbrook, A. Aspect, and G. V. Shlyapnikov, Phys. Rev. Lett. 89, 220406 (2002).

[18] F. Pereira dos Santos, J. Léonard, J. Wang, C. J. Barrelet, F. Perales, E. Rasel, C. S. Unnikrishnan, M. Leduc, and C. Cohen-Tannoudji, Eur. Phys. J. D 19, 103 (2002).

[19] Z.-C. Yan and J. F. Babb, Phys. Rev. A 58, 1247 (1998). 